\title{
Die Parteispende der Aktiengesellschaft
}

\author{
Von Wiss. Ass. Jakob Hahn
}

I. Einleitung

II. Die Parteispende im öffentlichen Recht

1. USA

2. Deutschland

a) Verfassungsrechtliche Vorgaben

b) Einfachgesetzliche Ausgestaltung

III. Die Parteispende in der aktienrechtlichen Kompetenzordnung

1. Die Spendenkompetenz de lege lata

a) Vorstand

aa) Unternehmensinteresse

bb) Unternehmensgegenstand

cc) Neutralitätspflicht

dd) Code of Conduct

b) Aufsichtsrat

c) Hauptversammlung

aa) Geschriebene Hauptversammlungskompetenz

bb) Ungeschriebene Hauptversammlungskompetenz

2. Die Spendenkompetenz de lege ferenda

a) Vereinigtes Königreich: s 366 Companies Act 2006

b) Eine Hauptversammlungskompetenz für die deutsche AG?

IV. Die Vorstandspflichten bei der Parteispende

1. Allgemeine Sorgfaltspflicht
a) Unternehmensinteresse
aa) Bezug zum Unternehmensgegenstand
bb) Gleichbehandlung politischer Parteien
cc) Konflikte mit der Parteiprogrammatik
b) Spendenhöhe
c) Unternehmensreputation

2. Legalitätspflicht
a) Parteiengesetz
b) Korruptionsstrafrecht

3. Treuepflicht

V. Fazit

Die Veröffentlichung dieses Artikels erfolgt gem. § 38 Abs. 4 UrhG. Am linken Seitenrand sind die entsprechenden Seitenzahlen aus der Printveröffentlichung angegeben. 


\title{
472 Die Parteispende der Aktiengesellschaft
}

\author{
Wiss. Ass. Jakob Hahn
}

Aktiengesellschaften zählen in Deutschland zu den bedeutendsten Spendern politischer Parteien. Der Beitrag nimmt dies zum Anlass, den rechtlichen Implikationen einer Parteispende durch Aktiengesellschaften auf den Grund zu gehen. Er befasst sich zunächst mit der öffentlich-rechtlichen Seite dieses Problemkomplexes und geht sodann den Fragen nach, wem im Kompetenzgefüge der Aktiengesellschaft die Entscheidung über Parteispenden obliegt und welche Pflichten den Vorstand bei der Vergabe von Parteispenden treffen. Dazu nimmt er auch die Rechtsentwicklung in den USA und dem Vereinigten Königreich in den Blick.

\section{Einleitung}

Aktiengesellschaften zählen regelmäßig zu den Großspendern deutscher Parteien. Ein Blick in deren aktuelle Rechenschaftsberichte zeigt, dass der großzügigste Einzelspender beider Volksparteien im Jahr 2015 jeweils eine Aktiengesellschaft war: bei der CDU die Deutsche Vermögensberatung AG mit einer Spende von $195.000 €$ und bei der SPD die Daimler AG mit einer Spende von $100.000 € .^{1}$

Auch wenn mittlerweile nahezu einhellig anerkannt ist, dass Parteispenden aus dem Gesellschaftsvermögen grundsätzlich zulässig sind, bietet es sich deshalb an, einige Facetten dieser Thematik zu vertiefen. Der Beitrag greift dazu drei Fragekomplexe auf. Vor dem Hintergrund der in den USA geführten Citizens United-Kontroverse leuchtet er zunächst aus, welche Rechte und Pflichten Partei und Spender im öffentlichen Recht treffen. Sodann analysiert er, wem im innergesellschaftlichen Kompetenzgefüge die Entscheidung über eine Parteispende obliegt, und unterzieht das Ergebnis einer rechtsvergleichenden und -politischen Würdigung. Abschließend werden die Einzelpflichten des Vorstands bei der Spendenvergabe ergründet.

\section{Die Parteispende im öffentlichen Recht}

\section{USA}

Wer sich mit den verfassungsrechtlichen Vorgaben für die Parteispende befasst, kommt nicht umhin, einen Blick in die Vereinigte Staaten zu werfen. Dort erlangte das Thema 2010 mit der Citizens United-Entscheidung des US Supreme Court großes Aufsehen. Antragstellerin in dem zugrundeliegenden Verfahren war eine konservative Nichtregierungsorganisation. Diese hatte eine kritische Dokumentation über Hillary Clinton produziert, die sie unmittelbar vor den Vorwahlen der Demokratischen Partei für die Präsidentschaftswahlen 2008 im Fernsehen ausstrahlen und öffentlich bewerben wollte. Dem stand Sec. 203 des Bipartisan Campaing Reform Act of $2002^{2}$ entgegen, der es Pub.L. 107-155, nach den federführenden Senatoren umgangssprachlich auch McCain-Feingold Act genannt. 
juristischen Personen untersagte „electioneering communications“ $\mathrm{zu}$ finanzieren, die weniger als 31 Tage vor Vorwahlen stattfinden. ${ }^{3}$

Unter Abkehr von seiner früheren Rechtsprechung ${ }^{4}$ entschied der Supreme Court mit der denkbar knappen Mehrheit von $5 \mathrm{zu} 4$ Stimmen, dass dieses Verbot die durch den 1. Zusatzartikel der US-Verfassung gewährleistete Meinungsfreiheit verletzt. Das Gericht war dabei entlang seiner ideologischen Trennlinie gespalten. Den Ausschlag für die Entscheidung gab der als swing voter bekannte Richter Anthony Kennedy, der die von der konservativen Seite unterstützte Mehrheitsmeinung verfasste. Er führte aus, dass es kategorisch ausgeschlossen sei, Einschränkungen der Meinungsfreiheit einzig an Eigenschaften des Äußernden zu knüpfen. Es sei dem Gesetzgeber darum verwehrt, bei der Meinungsfreiheit zwischen natürlichen und juristischen Personen zu diskriminieren. Demzufolge verletze jede Beschränkung der Wahlkampffinanzierung, die daran anknüpft, dass es sich bei dem Zuwender um eine juristische Person handelt, die Verfassung. ${ }^{5}$ Diese Haltung bekräftigte das Gericht in zwei Folgeentscheidungen. ${ }^{6}$

Diese Rechtsprechung erfuhr sowohl innerhalb des Gerichts als auch in der Rechtswissenschaft erhebliche Kritik. In einem Sondervotum zur Citizens United-Entscheidung, dem sich der gesamte liberale Flügel des Gerichts anschloss, bemängelte Richter John Paul Stevens, dass die Mehrheit die qualitativen Unterschiede zwischen natürlichen und juristischen Personen verkenne. Juristische Personen könnten weder wählen noch gewählt werden. Sie ließen sich durch Ausländer kontrollieren, weshalb ihre Interessen in Widerspruch zu denen der Wähler stehen könnten. Auch ihre finanziellen Möglichkeiten ließen besorgen, dass sie der Integrität und Fairness des demokratischen Prozesses schaden. Der Gesetzgeber sei darum berechtigt hiergegen Vorsorge zu treffen, wenn nicht gar dazu verpflichtet. ${ }^{7}$ Literaturstimmen werfen dem Gericht insbesondere vor, nicht ausreichend berücksichtigt zu haben, dass die Meinungsfreiheit auch im Lichte des Minderheitenschutzes zu betrachten sei. Juristische Personen seien aufgrund ihres Organisationsgrades und ihrer finanziellen Ausstattung natürlichen Personen strukturell überlegen und damit in der Lage, den Meinungsbildungsprozess im Vorfeld öffentlicher Wahlen einseitig zu ver-

473 zerren. ${ }^{8}$ Citizens United hat sich unlängst zu einem Reizwort für die politische Linke in den USA entwickelt. Allen voran Bernie Sanders fordert lautstark diese Rechtsprechung aufzugeben, da sie es Wohlhabenden erlaube, Wahlen zu kaufen. ${ }^{9}$

\section{Deutschland}

\section{a) Verfassungsrechtliche Vorgaben}

Hierzulande birgt das Thema deutlich weniger juristischen und politischen Sprengstoff als in den USA. Fest steht, dass der allgemeine Parteienfreiheit des Art. 21 Abs. 1 GG auch die Finanzierungsfreiheit umfasst. Parteien sind deshalb grundsätzlich befugt

$3 \quad$ Citizens United v. Federal Election Commission, 558 U.S. 310 (2010).

4 Austin v. Michigan Chamber of Commerce, 494 U.S. 652, 657-669 (1990); McConnell v. Federal Election Commission, 540 U.S. 93, 94-104 (2003).

$5 \quad$ Citizens United v. Federal Election Commission, 558 U.S. 310, 336-365 (2010).

6 Arizona Free Enterprise Club's Freedom Club PAC v. Bennett, 564 U.S. 721, 736-755 (2011); McCutcheon v. Federal Election Commission, 134 S. Ct. 1434, 1448-1462 (2014).

7 Citizens United v. Federal Election Commission, 558 U.S. 310, 394 (2010) (Stevens, J. dissenting). 8 Andre, 44 J. Marshall L. Rev. 69, 114-123 (2010-2011); Batchis, 36 N.Y.U. Rev. L. \& Soc. Change 5, 39-51 (2012); Sullivan, 124 Harv. L. Rev. 143, 146-155 (2010-2011).

9 Sanders, „Getting Big Money Out of Politics and Restoring Democracy“, abrufbar unter https://berniesanders.com/issues/money-in-politics/ (Abruf vom 8.6.2018). 
Spenden anzunehmen, ${ }^{10}$ müssen aber nach Art. 21 Abs. 1 Satz 4 GG über die Herkunft und Verwendung ihrer Mittel sowie über ihr Vermögen öffentlich Rechenschaft geben. Das spiegelbildliche Recht darauf, an Parteien zu spenden, hat das BVerfG bisher lediglich im Kontext der steuerlichen Abzugsfähigkeit am allgemeinen Gleichbehandlungsgrundsatz des Art. 3 Abs. 1 GG gemessen. ${ }^{11}$ Die Citizens United-Frage, ob sich der Spender auf die Meinungsfreiheit (Art. 5 Abs. 1 Satz 1 GG) berufen kann, ist im deutschen verfassungsrechtlichen Diskurs hingegen bislang wenig erörtert worden. Die wenigen Literaturstimmen, die sich mit dieser Frage auseinandersetzen, bejahen sie. So erblickten einige Autoren eine Verletzung der Meinungsfreiheit in einem durch eine Bundesratsinitiative des Landes Hessen 1959 vorgeschlagenen gesetzlichen Verbot der Parteispende durch juristische Personen. ${ }^{12}$ Andere meinen, der Spender könne sich gleich auf eine ganzes Bündel von Grundund grundrechtsgleichen Rechten berufen, zu denen neben der Meinungs- auch die Versammlungs-, Vereinigungs- und Parteiengründungsfreiheit sowie das aktive Wahlrecht zählten. ${ }^{13}$

Dem wird man insoweit zustimmen müssen, als dass die Spende an eine Partei zumindest dem Schutzbereich des Art. 5 Abs. 1 Satz 1 GG zuzuordnen ist. Diesem unterfallen Äußerungen, die durch das Element der Stellungnahme und des Dafürhaltens geprägt sind. ${ }^{14}$ Er umfasst damit auch Realakte, soweit diese als Teilnahme an einem Kommunikationsprozess und nicht als bloße soziale Interaktion gedeutet werden können. ${ }^{15}$ So verhält es sich mit der Parteispende, die sich gerade nicht in einer wirtschaftlichen Transaktion erschöpft, sondern darüber hinaus ein kommunikatives Element in sich trägt. Oder um es in den Worten des BVerfG zu fassen: „Der Bürger, der einer politischen Partei Geld spendet, bekennt sich damit in der Regel zu den Zielen dieser Partei, ähnlich wie wenn er ihr seine Wahlstimme geben würde". ${ }^{16}$

Handelt es sich bei dem Spender um eine Aktiengesellschaft, tritt die Besonderheit des Art. 19 Abs. 3 GG hinzu, wonach juristische Personen des Privatrechts lediglich Grundrechtsträger sind, soweit die Grundrechte ihrem Wesen nach auf sie anwendbar sind. Sie kann sich darum nicht auf Grundrechte berufen, die an Eigenschaften, Äußerungsformen oder Beziehungen anknüpfen, die nur natürlichen Personen wesenseigen sind. ${ }^{17}$ Hier klingen einem die mahnenden Worte des ehemaligen Verfassungsrichters Ernst-Wolfgang Böckenfördes in den Ohren, dem zufolge juristische Personen als solche keine politischen Mitwirkungsrechte hätten und insbesondere der Teilhabe an der politischen Willensbildung in der Form des aktiven und passiven Wahlrechts entbehrten. ${ }^{18}$ Das mag eine Differenzierung zwischen natürlichen und juristischen Personen bei der steuerlichen Abzugsfähigkeit von Parteispenden gebieten, worum es in Böckenfördes Sondervotum ging, dem sich später der gesamte 1. Senat unter Aufgabe

Klein in Maunz/Dürig, Loseblatt, Art. 21 GG Rz. 415; vgl. auch BVerfG v. 24.7.1979 - 2 BvF 1/78, BVerfGE 52, 63, 86. BVerfG v. 24.6.1958 - 2 BvF 1/57, BVerfGE 8, 51, 68 f.; BVerfG v. 14.6.1986 - 2 BvE 2/84, 2 BvR 442/84, BVerfGE 73, 40, 71.

Dübber, Parteienfinanzierung in Deutschland, 1962, S. 55 f.; Rüfner, AöR 89 (1964), 261, 292 (Fn. 123); sympathisierend Fleischer, AG 2001, 171, 179. Volkmann, JZ 2000, 539, 542; s. auch Morlok, 2. Aufl. 2013, § 25 ParteiG Rz. 1. BVerfG v. 22.6.1982 - 1 BvR 1376/79, BVerfGE 61, 1, 8; BVerfG v. 13.4.1994 - 1 BvR 23/94, BVerfGE 90, 241, 247; BVerfG v. 4.11.2009 - 1 BvR 2150/08, BVerfGE 124, 300, 320. Grabenwart in Maunz/Dürig, Loseblatt, Art. 5 GG Rz. 82. BVerfG v. 24.6.1958 - 2 BvF 1/57, BVerfGE 8, 51, 68; ähnlich BVerfG v. 3.12.1968 - 2 BvE 1/67, BVerfGE 24, 300, $360 \mathrm{f}$.

17 BVerfG v. 26.2.1997 - 1 BvR 2172/96, BVerfGE 95, 220, 242; BVerfG v. 9.10.2002 - 1 BvR 1611/96, 1 BvR 805/98, BVerfGE 106, 28, 42. 
seiner vormaligen Rechtsprechung anschließen sollte. ${ }^{19}$ Als personales Substrat ihrer Mitglieder lassen sich der Aktiengesellschaft Kommunikationsfreiheiten aber nicht pauschal absprechen. Dass sich auch juristische Personen des Privatrechts auf die Meinungsfreiheit berufen können, wird darum in Rechtsprechung und Literatur einhellig anerkannt. ${ }^{20}$

Die Aktiengesellschaft genießt folglich ein Artt. 5 Abs. 1 Satz 1, 19 Abs. 3 GG entspringendes subjektives Recht, an politische Parteien zu spenden. Dieses steht allerdings unter dem qualifizierten Gesetzesvorbehalt des Art. 5 Abs. 2 GG. Im Hinblick auf das konfligierende Recht des Bürgers auf gleiche Teilhabe an der politischen Willensbildung, welches im allgemeinen Gleichheitssatz und dem Demokratieprinzip wurzelt, ${ }^{21}$ ließen sich Beschränkung für das Spenderrecht der Aktiengesellschaft aber wohl rechtfertigen, zumal ihr grundrechtliches Schutzniveau gegenüber dem natürlicher Personen ohnehin abgeschwächt ist. ${ }^{22}$

\section{b) Einfachgesetzliche Ausgestaltung}

Als Parteispenden gelten nach $\S 27$ Abs. 1 Satz 3 ParteiG alle über Mitglieds- und Mandatsträgerbeiträge hinausgehenden Zahlungen. Die Dreh- und Angelnorm des Parteispendenrechts bildet $\S 25$ ParteiG. Die Vorschrift gewährleistet die Finanzierungsfreiheit der Parteien, indem sie deren grundsätzliches Recht zur Annahme von Spenden statuiert. Gleichzeitig unter-

474 wirft sie dieses Recht einer ganzen Reihe von Restriktionen. So schließt § 25 Abs. 2 ParteiG bestimmte Spenden von der Annahmebefugnis der Parteien aus. Dazu zählen u.a. Spenden von Unternehmen, die zu mehr als $25 \%$ im Eigentum der öffentlichen Hand stehen ( $§ 25$ Abs. 2 Nr. 5 ParteiG). Dieses Verbot verfolgt einen doppelten Schutzzweck: Es soll einerseits eine indirekte staatliche Parteienfinanzierung verhindern und damit sowohl die Gleichbehandlung der Parteien durch die öffentliche Hand, als auch die Einhaltung der absoluten Obergrenze der staatlichen Parteienfinanzierung ( $§ 18$ Abs. 2 Satz 1 ParteiG) gewährleisten. Andererseits soll es den Schutz der Parteien vor staatlicher Einflussnahme garantieren. ${ }^{23}$ Ebenso unzulässig sind Spenden, die der Partei erkennbar in Erwartung oder als Gegenleistung eines bestimmten wirtschaftlichen oder politischen Vorteils gewährt werden ( $§ 25$ Abs. 2 Nr. 7 ParteiG). Erhält eine Partei nach $\S 25$ Abs. 2 ParteiG unzulässige Spenden, hat sie diese unverzüglich an den Präsidenten des Deutschen Bundestages weiterzuleiten ( $\$ 25$ Abs. 4 ParteiG).

Ferner treffen die Parteien ab einer gewissen Spendenhöhe Offenlegungspflichten. Dafür erlangen zwei Schwellenwerte Bedeutung: Übersteigt der Gesamtwert einer Spende in einem Kalenderjahr $10.000 €$, ist sie unter Angabe des Namens und der Anschrift des Zuwenders sowie ihrer Gesamthöhe im Rechenschaftsbericht zu verzeichnen (§ 25 Abs. 3 Satz 1 ParteiG). Übersteigt eine Spende im Einzelfall die Höhe von 50.000 $€$, ist sie unverzüglich dem Präsidenten des Deutschen Bundestages anzuzeigen, der

BVerfG v. 9.4.1992 - 2 BvE 2/89, BVerfGE 85, 264, 315 ff.

BVerfG v. 6.11.1968 - 1 BvR 501/62, BVerfGE 24, 278, 282; BVerfG v. 16.6.2000 - 1 BvR 1539/94, NVwZ 2000, 1281, 1282; P. M. Huber in v. Mangoldt/Klein/Starck, 7. Aufl. 2018, Art. 19 GG Rz. 320; Remmert in Maunz/Dürig, Loseblatt, Art. 19 Abs. 3 GG Rz. 102; Sachs in Sachs, 8. Aufl. 2018, Art. 19 GG Rz. 74.

BVerfG v. 24.6.1958 - 2 BvF 1/57, BVerfGE 8, 51, 68 f.; BVerfG v. 24.6.1979 - 2 BvF 1/78, BVerfGE 52, 63, 88; BVerfG v. 14.6.1986 - 2 BvE 2/84, 2 BvR 442/84, BVerfGE 73, 40, 71; BVerfG v. 9.4.1992 - 2 BvE 2/89, BVerfGE 85, 264, 315 ff.

Volkmann, JZ 2000, 539, $542 \mathrm{f}$.

Lampe in Erbs/Kohlhaas, Strafrechtliche Nebengesetze, Loseblatt, § 25 ParteiG Rz. 15; Morlok, 2. Aufl. 2013, § 25 ParteiG Rz. 6. 
diese zeitnahe als Bundestagsdrucksache zu veröffentlichen hat (§ 25 Abs. 3 Satz 2 f. ParteiG).

Verstöße gegen die Annahmeverbote und Veröffentlichungspflichten sanktioniert $\S 31 \mathrm{c}$ ParteiG mit Zahlungspflichten: Nimmt eine Partei entgegen § 25 Abs. 2 ParteiG eine unzulässige Spende an, entsteht gegen sie demnach ein Anspruch in dreifacher Höhe. Veröffentlicht sie Spenden entgegen § 25 Abs. 3 ParteiG nicht im Rechenschaftsbericht, entsteht gegen sie ein Anspruch in doppelter Höhe.

\section{Die Parteispende in der aktienrechtlichen Kompetenzordnung}

\section{Die Spendenkompetenz de lege lata}

Die Fähigkeit des Vorstands, im Außenverhältnis wirksam im Namen der Gesellschaft Zuwendungen an politische Parteien zu tätigen, folgt aus seiner organschaftlichen Vertretungsmacht ( $\$ 78$ Abs. 1 Satz 1 AktG). Fraglich ist allein, wem im Binnenverhältnis die Entscheidung hierüber zusteht.

\section{a) Vorstand}

Die Schlacht um die Frage, ob der Vorstand grundsätzlich befugt ist, Parteispenden aus dem Gesellschaftsvermögen zu tätigen, ist heute als geschlagen anzusehen. Ihr Verlauf soll hier deshalb nur der Vollständigkeit halber wiedergegeben werden.

\section{aa) Unternehmensinteresse}

Bei der Leitung der Gesellschaft ( $§ 76$ Abs. 1 AktG) hat der Vorstand dem Unternehmensinteresse Rechnung zu tragen. In der Frage nach dem Inhalt dieses Interesses scheiden sich bekanntlich die Geister: Die herrschende Meinung verfolgt einen interessenpluralistischen Ansatz, der die Belange von Aktionären, Arbeitnehmern, Gläubigern und der Allgemeinheit gleichermaßen berücksichtigt (stakeholder value), ${ }^{24}$ eine andere Ansicht möchte Aktionärsinteressen einen Gewichtsvorsprung gewähren (moderater shareholder value $)^{25}$. Mit beiden Auffassungen gelangt man allerdings zu demselben Ergebnis: (Partei)Spenden aus dem Gesellschaftsvermögen sind grundsätzlich zulässig, da sie die soziale Akzeptanz der Gesellschaft fördern und damit langfristig ihre Rentabilität sichern. ${ }^{26}$

Für den Sonderbereich der Parteispenden sieht sich diese Begründung Anfechtungen ausgesetzt: Anders als bei allgemeinen Spenden, die aus gemeinnützigen, wohltätigen Motiven erfolgten, erfüllten Parteispenden keine sozialpolitische Erwartung der Allgemeinheit. Ob ihrer fehlenden Transparenz lösten sie eher Unbehagen in der Öffentlichkeit aus. ${ }^{27}$ Dieses pessimistische Bild der Parteispende verkennt allerdings die essentielle Rolle, die Parteien in der repräsentativen Demokratie einnehmen. Parteien stellen ein verfassungsrechtlich notwendiges Instrument der Willensbildung des Volkes 
dar, weshalb ihnen auch Art. 21 GG einen besonderen Status einräumt. ${ }^{28}$ Es besteht deshalb sehr wohl ein Interesse der Allgemeinheit daran, dass die Parteien über ausreichende finanzielle Mittel verfügen, um ihre verfassungsrechtliche Rolle auszufüllen. Damit gilt auch für Parteispenden, dass sie geeignet sind, die soziale Akzeptanz der Gesellschaft zu fördern. ${ }^{29}$ Auch mangelnde Transparenz wird man Parteispenden nicht attestieren können, sind Parteien doch sogar Adressat besonderer Offenlegungspflichten (§ 25 Abs. 3 ParteiG). Die besondere Konfliktträchtigkeit von Parteispenden schließt sie darum nicht pauschal vom Unternehmensinteresse aus. Vielmehr hat der Vorstand dieser bei der Ausübung seines Leitungsermessens Rechnung zu tragen. ${ }^{30}$

\section{bb) Unternehmensgegenstand}

Gegen eine Vorstandskompetenz für Parteispenden ist vereinzelt eine Schranke aus dem Unternehmensgegenstand abgeleitet worden. Bei Spenden handele es sich grundsätzlich um unternehmensfremde Aufwendungen. Als treuhänderischer Verwalter fremden Vermögens sei der Vorstand deshalb nur berechtigt sie zu tätigen, wenn ihnen die Umschreibung des Unternehmensgegenstandes in der Satzung ausdrücklich hierzu ermächtige. $^{31}$

Diese Auffassung stellt allerdings überspannte Anforderung an die satzungsmäßige Konkretisierung des Unternehmensgegenstandes. Der Unternehmensgegenstand gibt bloß die Art der

Tätigkeit wieder, welche die Gesellschaft zu betreiben beabsichtigt. ${ }^{32}$ Mit welchen Mitteln diese Tätigkeit verfolgt wird, hat hingegen der Vorstand im Rahmen seines Ermessens zu bestimmen. Parteispenden schließt der Unternehmensgegenstand darum, auch ohne ihre ausdrückliche Erwähnung in der Satzung, nicht von vornherein aus. ${ }^{33}$

\section{cc) Neutralitätspflicht}

Im älteren Schrifttum wurde die These aufgestellt, der Vorstand sei zur parteipolitischen Neutralität verpflichtet, da die Gesellschaft politisch neutral sei. ${ }^{34}$ Für diese Prämisse blieben ihre Vertreter allerdings eine Begründung schuldig. Aus dem öffentlichen Recht ergibt sich eine Pflicht der Gesellschaft zur parteipolitischen Neutralität nicht, genießt sie doch im Gegenteil gar von Verfassung wegen ein subjektives Recht, sich mittels Parteispenden am öffentlichen Meinungskampf zu beteiligen. ${ }^{35}$

Ebenso vermisst man eine tragfähige Herleitung einer solchen Pflicht aus dem Gesellschaftsrecht, ${ }^{36}$ kann doch der Verbandszweck auch politischer Natur sein. ${ }^{37}$ Und selbst dort, wo er sich in einer erwerbswirtschaftlichen Betätigung erschöpft, folgt aus ihm nicht die politische Neutralität der Gesellschaft. Bereits im Ausgangspunkt stellt

BVerfG v. 19.6.1966 - 2 BvF 1/65, BVerfGE 20, 56, 100 f.; BVerfG v. 9.4.1992 - 2 BvE 2/89, BVerfGE 85, 264, 286. 2010, § 76 AktG Rz. 40; Kort in Großkomm/ AktG, 5. Aufl. 2015, § 76 AktG Rz. 110. 
die erwerbswirtschaftliche Betätigung nämlich keine (interessen-)neutrale Tätigkeit dar, sondern ist durch das Unternehmensinteresse geleitet. Wie soeben gezeigt, lässt sich auf dieses aber ein Verbot von Parteispenden gerade nicht stützen. Weder die Gesellschaft noch der Vorstand sind deshalb zur politischen Neutralität verpflichtet.

\section{dd) Code of Conduct}

In der Praxis verbietet allerdings häufig ein sog. „Code of Conduct" Spenden aus dem Gesellschaftsvermögen an politische Parteien. Einer Untersuchung aus dem Jahr 2014 zufolge erlegt sich eine wachsende Zahl bedeutender Aktiengesellschaften eine Selbstbeschränkung auf. ${ }^{38}$ Soweit ein derartiger "Code of Conduct" existiert und er Rechtsqualität besitzt, ist der Vorstand im Binnenverhältnis verpflichtet, von Parteispenden abzusehen.

\section{b) Aufsichtsrat}

Der Aufsichtsrat ist zwar nicht zur Geschäftsführung befugt (§ 111 Abs. 4 Satz 1 AktG), kann aber beschließen, dass bestimmte Geschäfte von seiner Zustimmung abhängen (§ 111 Abs. 4 Satz 2 AktG). Es ist grundsätzlich möglich, Parteispenden in diesen Zustimmungskatalog aufzunehmen, ${ }^{39}$ und erscheint überdies auch sinnvoll, da sich durch diesen Kontrollmechanismus der Eindruck vermeiden ließe, der Vorstand könne bei der Spendenvergabe eigene politische Präferenzen vor das Unternehmensinteresse stellen. ${ }^{40}$ Aus der normativen Konzeption des $\S \S 76$ Abs. 1, 111 Abs. 4 Satz 1 AktG ergibt sich jedoch, dass Maßnahmen des gewöhnlichen Geschäftsbetrieb dem Vorstand obliegen und die Aufsichtsratskompetenz Ausnahmecharakter hat. Allein Maßnahmen, die für die Gesellschaft von besonderer Bedeutung sind, insbesondere weil sie die Vermögens-, Finanz- oder Ertragslage grundlegend verändern, dürfen deshalb den Gegenstand eines Zustimmungsvorbehalts bilden. ${ }^{41}$ Eine Zustimmungskompetenz für Parteispenden kann sich der Aufsichtsrat deshalb erst ab einer außergewöhnlichen Höhe schaffen. ${ }^{42}$ In der Praxis hat sich dies wohl (noch) nicht durchgesetzt, soweit man den gängigen Formularbüchern eine Indizwirkung beimisst. ${ }^{43}$

\section{c) Hauptversammlung}

\section{aa) Geschriebene Hauptversammlungskompetenz}

Eine Literaturansicht erblickt eine Zuständigkeit der Hauptversammlung für Parteispenden aus dem Gesellschaftsvermögen de lege lata in $\S 174$ Abs. 1 Satz 1 AktG. Danach beschließt die Hauptversammlung über die Verwendung des Bilanzgewinns. Zwar würden Parteispenden als Aufwendungen den Bilanzgewinn nach dem maßgeblichen festgestellten Jahresabschluss ( $§ 174$ Abs. 1 Satz 2 AktG) mindern. Es sei aber eine materielle Betrachtung geboten, derzufolge auch die Spendenvergabe bereits eine Form der Gewinnverwendung darstellt. Dies folge aus dem Telos des $\S 58$ Abs. 3 Satz 2 AktG, nachdem eine anderweitige Gewinnverwendung (als die Rücklagenbildung, der Vortrag 
oder die Verteilung) einem Beschluss der Hauptversammlung vorbehalten wären. Wie die Gesetzesmaterialien demonstrierten, fielen hierunter auch Zuwendungen an eine gemeinnützige Anstalt. ${ }^{44}$ Dem seien Parteispenden gleichzustellen. ${ }^{45}$

Diese Auffassung konnte sich zu Recht nicht durchsetzen. Wie auch ihre Vertreter im Ausgangspunkt richtig erkannt haben, bilden Parteispenden einen Teil der Erfolgsberechnung und wirken sich bilanziell als sonstige Aufwendungen gewinnschmälernd aus. Prima facie sind Parteispenden damit der Feststellung des Jahresabschlusses vorgeschaltet und stellen keine Verwendung des Bilanzgewinns dar. ${ }^{46}$ Eine davon abweichende „materielle" Interpretation des Tatbestandsmerkmals lässt sich schon mit dem Wortlaut des $\S 179$ Abs. 1 Satz 1 kaum vereinbaren: „Bilanzgewinn“ kann schwerlich etwas meinen, was bilanzrechtlich kein Gewinn ist. Eine solche Abweichung von den formalen Regeln der Bilanzierung würde überdies erhebliche Rechtsunsicherheiten schaffen, provoziert sie doch notwendig die Anschlussfrage, was entgegen dem Bilanzrecht noch „materiell“ Bilanzgewinn darstellt. ${ }^{47}$

Der gewichtigste Einwand gegen diese Ansicht lautet jedoch, dass er einer Legitimation entbehrt. Der Rekurs auf $\S 58$ Abs. 3 Satz 2 AktG erweist sich schon deshalb als untauglich, da er keine geborene Hauptversammlungszuständigkeit schafft, sondern eine Satzungsermächtigung voraussetzt. ${ }^{48}$ Die Heranziehung seines Telos droht zudem Gefahr, in einem Zirkelschluss zu enden. Der Wortlaut der Norm setzt den Begriff des Bilanzgewinns bereits voraus und knüpft an ihn lediglich Rechtsfolgen. Anhaltspunkte für seine Definition bietet er nicht. Nicht anders verhält es sich auch mit der Gesetzesbegründung. Sie besagt nicht, dass Zuwendungen an gemeinnützige Anstalten stets einen Gewinnverwendungsbeschluss der Hauptversammlung voraussetzen, sondern, dass ein Gewinnverwendungsbeschluss, durch den eine solche Zuwendung erfolgt, eine entsprechende Satzungsermächtigung benötigt. Eine Hauptversammlungszuständigkeit für Parteispenden ergibt sich darum nicht aus $\S 179$ Abs. 1 Satz 1 AktG.

\section{bb) Ungeschriebene Hauptversammlungskompetenz}

Eine ungeschriebene Hauptversammlungskompetenz für Parteispenden nach der höchstrichterlichen Holzmüller/GelatineDoktrin ${ }^{49}$, ist von vornherein hypothetischer Natur. Von der quantitativen $80 \%$-Schwelle ${ }^{50}$ liegen Spendenbeträge in der Praxis weit entfernt, gleichviel an welche der diskutierten Kennziffern ${ }^{51}$ man dafür anknüpft. Um eine Holzmüller-Maßnahme handelt es sich bei Parteispenden deshalb nicht, auch wenn

Siehe Kropff, Aktiengesetz, 1965, S. 78: „Will die Hauptversammlung im Gewinnverwendungsbeschluß den Bilanzgewinn weder in Rücklage stellen noch unter die Aktionäre verteilen, sondern einem Dritten, etwa einer gemeinnützigen Anstalt, zuwenden, so bedarf sie hierzu einer Ermächtigung in der Satzung (...).“

Vgl. zu allem Meilicke, NJW 1959, 409, 410 ff.; Kulitz, Unternehmerspenden an politische Parteien, 1983, S. $165 \mathrm{ff}$.

Fleischer, AG 2001, 171, 177; Mertens in FS Goerdeler, 1987, S. 349, 352; Uwe H. Schneider, AG 1983, 205, 212; Windmöller in FS Budde, 1995, S. 675, 677; s. auch zum Schweizer Recht Jutzi, recht 2017, 251, 261.

$47 \quad$ Vgl. Mertens in FS Goerdeler, 1987, S. 349, 359.

$48 \quad$ Fleischer, AG 2001, 171, 177; Mertens in FS Goerdeler, 1987, S. 349, 358.

49 BGH v. 25.2.1982 - II ZR 174/80, BGHZ 83, 122, 131 ff. = AG 1982, 158, 162 f.; BGH v. 26.4.2004 - II ZR 155/02, BGHZ 159, 30, $37 \mathrm{ff} .=$ AG 2004, 384, $386 \mathrm{ff}$.

$50 \quad$ Vgl. BGH v. 26.4.2004 - II ZR 155/02, BGHZ 159, 30, 45 = AG 2004, 384, 388.

51 Dazu Kubis in MünchKomm/AktG, 4. Aufl. 2018, § 119 AktG Rz. 50. 
man den qualitativ erforderlichen Mediatisierungseffekt bei der kompensationslosen Fortgabe von Gesellschaftsvermögen wohl annehmen könnte. ${ }^{52}$

\section{Die Spendenkompetenz de lege ferenda}

Im Regelfall obliegt es damit dem Vorstand, allein über Parteispenden aus dem Gesellschaftsvermögen zu entscheiden. Einen anderen Weg beschreitet das Recht des Vereinigten Königreichs, was auch für Reformüberlegungen zum deutschen Recht Anstoß geben könnte.

\section{a) Vereinigtes Königreich: s 366 Companies Act 2006}

Nach s 366 Companies Act 2006 dürfen Gesellschaften politischen Parteien keine Spenden gewähren, es sei denn, die Gesellschafter haben hierin durch Beschluss eingewilligt. Handelt es sich bei der Gesellschaft um eine Konzerntochter, so ist ein Beschluss der Gesellschafter der Konzernmutter erforderlich. Den Beschlussgegenstand bildet allerdings allein die Frage, ob die Gesellschaft überhaupt an politische Parteien spenden soll. Spezifische Vorgaben darüber, an welche Parteien zu spenden ist, kann er nicht machen. Er hat ferner einen Höchstbetrag festzulegen, der für den Zeitraum seiner Wirksamkeit an Spenden vergeben werden darf (s 367 Companies Act 2006). Ist nichts anderes bestimmt, gilt der Beschluss für vier Jahre (s 368 Companies Act 2006). Die Wirksamkeit der Zuwendung im Außenverhältnis bleibt von einer fehlenden Zustimmung der Gesellschafter unberührt. Vergibt der Vorstand eine Parteispende, ohne den erforderlichen Beschluss eingeholt zu haben, haftet er der Gesellschaft hierfür auf Schadensersatz. ${ }^{53}$

Die Regelung geht auf eine Empfehlung des Neill Committee zurück, das Ende der 90er-Jahre nach mehreren Spendenskandalen eingesetzt worden war, um eine umfassende Reform des Parteispendenrechts im Vereinigten Königreich zu entwickeln. ${ }^{54} \mathrm{Sie}$ verfolgt einen doppelten Schutzzweck: Einerseits sei die Entscheidung, öffentlich eine bestimmte politische Partei zu unterstützen, von einer solchen materiellen Tragweite für die Gesellschaft, dass sie das Management nicht allein fällen könnte, sondern einen Beschluss der Gesellschafter erfordere. Andererseits soll sie die Spendentransparenz erhöhen, indem die Identität desjenigen, der wirtschaftlich gesehen Spender ist, offengelegt wird. ${ }^{55}$

Beide Begründungsstränge müssen sich Kritik aus dem Schrifttum gefallen lassen. So gewähre s 366 Companies Act 2006 den Gesellschaftern nur ein erheblich eingeschränktes Mitbestimmungsrecht: Sie dürfen den Vorstand allein auf Vorrat dazu ermächtigen, überhaupt Spenden an politische Parteien zu vergeben, können aber nicht festlegen, welche Partei konkret begünstigt wird. ${ }^{56}$ Um die Spendentransparenz zu erhöhen, sei die Norm schon ihrer Struktur nach untauglich. Denn die Kompetenz der Gesellschafterversammlung zwinge gar nicht dazu, die Identität desjenigen 308, 309. https://assets.publishing.service.gov.uk/government/uploads/system/uploads/attachment_data/ file/336870/5thInquiry_FullReport.pdf (Abruf vom 8.6.2018). 
preiszugeben, der wirtschaftlich hinter der Spende stehe. Es bliebe insbesondere bei den zahlreichen inhabergeführten Gesellschaften weiterhin möglich, diese zu verschleiern, indem die Gesellschaft als Strohmann eingeschaltet werde. ${ }^{57}$ In der Praxis sei überdies zu beobachten, dass Manager lieber ganz davon absehen, Parteispenden aus dem Gesellschaftsvermögen zu gewähren, statt die Zustimmung der Gesellschafterversammlung einzuholen. ${ }^{58}$

\section{b) Eine Hauptversammlungskompetenz für die deutsche AG?}

Ist eine s 366 Companies Act 2006 entsprechende Regelung für das deutsche Aktienrecht erstrebenswert? Diese Frage berührt ein konfliktträchtiges Ewigkeitsthema der modernen Aktiengesellschaft: die nach der „richtigen“ Kompetenzverteilung zwischen Vorstand und Hauptversammlung. Ihre Beantwortung will wohl überlegt sein, operiert man doch am offenen Herzen des aktienrechtlichen Kompetenzgefüges. Sie kann hier deshalb nicht abschließend erfolgen. Vielmehr sollen einige ausgewählte Argumente skizziert werden.

Sucht man nach einer materiellen Rechtfertigung für eine Hauptversammlungskompetenz, so wäre an die erste Ratio des s 366 Companies Act 2006 anzuknüpfen, wonach die Tragweite der Entscheidung, sich in die Parteipolitik einzumischen, nach einer Beteiligung der Aktionäre verlange. Parteispenden sind ein in besonderer Weise konfliktträchtiges Thema. Das gilt nicht nur für die Außenbeziehungen der Gesellschaft, sondern auch für das Innenverhältnis. Die Aktionäre haben möglicherweise unterschiedliche politische Präferenzen, die sich wiederum nicht zwingend mit denen des Vorstands decken. Es gilt also (auch) eine klassische Agenturproblematik zu bewältigen: den potentiellen Interessenkonflikt zwischen dem Vorstand, als Verwalter fremder Mittel, und den Aktionären. ${ }^{59}$

Der rechtstatsächliche Befund zeigt, dass diese Konflikte in einer wachsenden Zahl an Gesellschaften als so schwerwiegend angesehen werden, dass man es vorzieht, ihnen ganz aus dem Weg zu gehen, indem man auf Parteispenden in einem Code of Conduct vollständig verzichtet. ${ }^{60}$ Dass sich diese radikale Lösung aus Sicht der Allgemeinheit als wünschenswert darstellt, muss bezweifelt werden, ist doch die Demokratie auf die Partei und die Partei auf eine ausreichende Finanzierung angewiesen.

Die Hauptversammlung könnte ein geeignetes Forum, um diese Konflikte ohne einen totalen Spendenverzicht zu bewältigen, da sie die Spende auf breiter (aktionärs-)demokratischer Basis legitimieren kann. Dazu müsste man ihr allerdings weitreichendere Befugnisse als s 366 Companies Act 2006 gewähren und sie dazu ermächtigen, nicht nur über das "Ob“ von Parteispenden generell zu entscheiden, sondern auch das „Wie“ (begünstigte Partei, Höhe) näher zu konkretisieren. Der hierzu erforderliche Sachverstand lässt sich der Hauptversammlung nicht pauschal absprechen. ${ }^{61}$ Auch erweist sich der Delegationsvorteil bei Parteispenden als deutlich geringer als bei anderen unternehmerischen Entscheidungen, da sie keine unverzüglichen Reaktionen der Gesellschaft im Tagesgeschäft erfordern. Dies gilt umso mehr, wenn man der Hauptversammlung nicht die Letztentscheidung über die Parteispende überantwortet, sondern lediglich nach einer Vorratsermächtigung für den Vorstand verlangt. ${ }^{62}$

Williams, (2012) 75 Modern Law Review 951, 968-970.

Davies/Worthington, Gower's Principles of Modern Company Law, 10. Aufl. 2016, Kap. 16 Rz. 85. Vgl. H. P. Westermann, ZIP 1990, 771, 774 f.; Williams, (2012) 75 Modern Law Review 951, 957961.

60 Siehe III.1.a)dd).

61 So aber Mertens in FS Goerdeler, 1987, S. 349, 359; Mertens/Cahn in KölnKomm/AktG, 3. Aufl. 2010, § 76 AktG Rz. 40. 
Mit dieser Konzeption lässt sich auch einem weiteren Einwand gegen eine Hauptversammlungszuständigkeit begegnen: Diese könne einen Minderheitenschutz für opponierenden Aktionäre gar nicht gewährleisten, da sie per Mehrheitsbeschluss entscheide. Der Vorstand dürfte hingegen im Regelfall auf eine ausgewogenere Spendenvergabe achten. ${ }^{63}$ Überlasst man aber die Entscheidung über eine konkrete Spende dem Vorstand, der sich dabei nur im Rahmen einer Ermächtigung durch die Hauptversammlung zu bewegen hat, kann dieser auch dem Minderheitenschutz Rechnung tragen.

Kritisch wird man jedoch die rechtstatsächlichen Erfahrungen aus dem Vereinigten Königreich berücksichtigen müssen. Sollte sich bewahrheiten, dass das Management aus Scheu vor einer Auseinandersetzung in der Gesellschafterversammlung lieber gleich darauf verzichtet, überhaupt Parteispenden zu tätigen, wäre durch die Einführung einer Hauptversammlungskompetenz wenig gewonnen.

\section{Die Vorstandspflichten bei der Parteispende}

Auch wenn der Vorstand grundsätzlich berechtigt ist, Parteispenden aus dem Gesellschaftsvermögen zu tätigen, unterliegt er dabei einigen Schranken, die sich aus seinem aktienrechtlichen Pflichtenprogramm (§93 Abs. 1 AktG) ergeben. Missachtet er diese, drohen ihm nicht nur Schadensersatzansprüche der Gesellschaft und eine Abberufung, sondern unter Umständen auch eine Untreuestrafbarkeit (§ $266 \mathrm{StGB})^{64}$.

\section{Allgemeine Sorgfaltspflicht}

Parteispenden stellen eine unternehmerische Entscheidung dar. Entsprechend genießt der Vorstand aufgrund der Business Judgement Rule (§ 93 Abs. 1 Satz 2 AktG) bei ihrer Vergabe einen unternehmerischen Ermessensspielraum. ${ }^{65}$ Bei seiner Ermessensentscheidung hat der Vorstand eine Prognose anzustellen, bei der er mögliche Vor- und Nachteile der Spende gegeneinander abzuwägen hat. ${ }^{66}$ Im Folgenden sollen einige Einzelaspekte konkretisiert werden, die es dabei zu berücksichtigen gilt.

\section{a) Unternehmensinteresse}

Auch wenn es das Unternehmensinteresse dem Vorstand nicht per se verwehrt, an politische Parteien zu spenden, können sich aus ihm bei der konkreten Spendenentscheidung gleichwohl Schranken ergeben.

\section{aa) Bezug zum Unternehmensgegenstand}

So wird in der Literatur verlangt, Spenden aus dem Gesellschaftsvermögen müssten allgemein einen - wenn vielleicht auch losen - Bezug zum Unternehmensgegenstand aufweisen. ${ }^{67}$ Gerade bei Parteispenden wäre es aber äußerst bedenklich, wenn der Vorstand sie nur in Erwartung eines bestimmten Vorteils für die Gesellschaft tätigte. Solch ein Verhalten über- 
schreitet schnell die Schwelle zur strafrechtlichen Relevanz, ${ }^{68}$ zumindest aber entstünde in der Öffentlichkeit leicht der Eindruck, der Vorstand versuche Politiker zu kaufen. ${ }^{69}$ Bei Spenden für soziale, wissenschaftliche und kulturelle Zwecke mag ein konkreter Bezug zum Unternehmensgegenstand, dem Vorstand einen größeren Handlungsspielraum eröffnen. ${ }^{70}$ Bei Parteispenden wirft er demgegenüber eher Compliance-Fragen auf.

\section{bb) Gleichbehandlung politischer Parteien}

Die vorgenannte Ansicht findet ihren Antagonisten in einer Literaturmeinung, der zufolge der Vorstand Parteien entsprechend ihrer politischen Bedeutung bei der Spendenvergabe gleichbehandeln solle. Bevorzuge der Vorstand einseitig eine bestimmte Partei, könne sich das negativ auf Sozialprestige der Gesellschaft auswirken. ${ }^{71}$

Man mag dem als allgemeine Handlungsempfehlung folgen, um Unmut politisch anders denkender Aktionäre und Dritter zu vermeiden. ${ }^{72}$ Als Rechtspflicht des Vorstandes lässt sich solch ein Gleichbehandlungsgebot jedoch nicht konstruieren. Das Gebot, Parteien gleichzubehandeln, richtet sich an den Staat ( $\$ 5$ ParteiG), nicht aber an private Akteure. Es kann sogar im wohlverstandenen Interesse der Gesellschaft sein, bei der Spendenvergabe solche Parteien zu bevorzugen, deren Programmatik ihren unternehmerischen Zielen eher entspricht. Wenn die Gesellschaft entsprechend ihrer Präferenzen bei der Spendenvergabe diskriminiert, übt sie ihre grundrechtlich gewährleistete Spenderfreiheit aus. Es lässt sich dann aber nicht begründen, dass der Vorstand im Innenverhältnis verpflichtet sei, zu vermeiden, dass genau dieser Eindruck entsteht.

\section{cc) Konflikte mit der Parteiprogrammatik}

Probleme ergeben sich vielmehr für den Fall, dass der Vorstand eine Partei fördert, deren Programmatik im Widerspruch zu den wirtschaftlichen Interessen der Gesellschaft steht. Beispiele hierzu lassen sich leicht bilden, etwa das eines Rüstungsunternehmens und der Partei, die für ein Verbot von Rüstungsexporten eintritt. Mit einem ähnlichen Fall hatte sich bereits das OLG Hamburg im Kontext der Abberufung eines Aufsichtsratsmitglieds aus wichtigem Grund zu befassen, als der damalige schleswig-holsteinische Minister Günther Jansen in den Aufsichtsrat der HEW AG berufen worden war. Jansen trat entschieden für einen Ausstieg aus der Atomkraft ein; die HEW betrieb mehrere Kernkraftwerke. Das OLG Hamburg nahm einen wichtigen Grund an, da Jansens politische Agenda für die Gesellschaft Milliardenverluste zur Folge hätte und damit in einem krassen Widerspruch zum Unternehmensinteresse stehe. ${ }^{73}$

In solchen Sachverhaltslagen würde sich auch der Vorstand pflichtwidrig verhalten, wenn er eine Partei, deren Programmatik mit dem Unternehmensinteresse schlechterdings unvereinbar ist, durch Spenden förderte. Abseits derartiger Evidenzfälle, lassen sich jedoch viele Konstellationen vorstellen, in denen der Widerspruch von Unternehmensinteresse und Parteiprogrammatik weniger deutlich zutage tritt: Darf der Vorstand eines Automobilherstellers an eine Partei spenden, die sich dafür einsetzt, dass in

Siehe IV.2.b).

Bayer/Hoffmann, AG 2014, R371, R374; Götze/Bicker in Krieger/Uwe H. Schneider, Hdb. Managerhaftung, 3. Aufl. 2017, § 30 Rz. 107; Kind, NZG 2000, 567, 569.

Fleischer, AG 2001, 171, 178.

Kind, NZG 2000, 567, 569 f.; verhaltener Mertens/Cahn in KölnKomm/ AktG, 3. Aufl. 2010, § 76 AktG Rz. 41: „Maß und Ausgewogenheit“".

So verfährt etwa die Daimler AG, siehe „Daimler spendet 320000 Euro“, FAZ, 8.5.2018, S. 19: „Jeweils 100000 Euro erhalten die CDU und die SPD, jeweils 40000 Euro bekommen die CSU, die FDP und die Grünen.“

OLG Hamburg v. 23.1.1990 - 11 W 92/89, AG 1990, 218 ff. 
einigen Jahrzehnten keine Kraftfahrzeuge mit Verbrennungsmotoren mehr zugelassen werden, wenn das Unternehmen selbst alternative Antriebstechniken entwickelt? Hier ist in Erinnerung zu rufen, dass entsprechend dem pluralistischen Charakter des Unternehmensinteresses, nicht alles, was der Gesellschaft auf kurze Sicht wirtschaftlich nachteilig ist, auch im Widerspruch zum Unternehmensinteresse stehen muss. Man wird von daher dem Vorstand eine Einschätzungsprärogative gewähren müssen und eine Sorgfaltspflichtverletzung erst dann annehmen können, wenn der Konflikt zwischen Unternehmensinteresse und Parteiprogrammatik unauflösbar ist.

\section{b) Spendenhöhe}

Es besteht Einigkeit, dass der Vorstand Spenden aus dem Gesellschaftsvermögen nur in angemessener Höhe tätigen darf. ${ }^{74}$ Weniger klar umrissen ist hingegen, wonach sich die angemessene Höhe einer Spende bestimmen lässt. Die steuerliche Abziehbarkeit, die eine Literaturmeinung als Grenze vorschlug, ${ }^{75}$ vermag keine Orientierung zu bieten, da Kapitalgesellschaften Parteispenden nicht mehr als Betriebsausgaben absetzen können ( 8 Abs. 1 Satz $1 \mathrm{KStG}, \S 4$ Abs. $6 \mathrm{EStG}$ ).

Die Angemessenheit der Spende lässt sich richtigerweise nur in Relation zu ihrer Kapitalausstattung, Finanzierung und Ertragslage beurteilen. ${ }^{76}$ Dabei ist die gesamte wirtschaftliche Situation der Gesellschaft zu beurteilen. Gegriffene Größen wie $1 \%{ }^{77}$ oder $5 \%$ des Bilanzgewinns, ${ }^{78}$ wie sie gelegentlich vorgeschlagen werden, erweisen sich daher als zu starr. ${ }^{79}$ Es kommt vielmehr darauf an, dass der Gesellschaft ausreichend Liquidität verbleibt, um im Allgemeinen langfristig rentabel zu wirtschaften, und im Besonderen ihre Verbindlichkeiten zu bedienen und in Zukunft erforderliche Investitionen zu tätigen. Subsidiär dazu kann auch der Aspekt der Verkehrsüblichkeit herangezogen werden. ${ }^{80}$

\section{c) Unternehmensreputation}

Die Unternehmensreputation bildet einen vergleichsweise jungen Topos im Katalog der Vorstandspflichten. Sie wird in der Debatte meist vorgebracht, um einen Ermessensspielraum des

Vorstands bei Handlungen zu begründen, die der Gesellschaft finanziell unmittelbar nachteilig sind - etwa wenn er davon absieht, von einer legalen, aber als aggressiv verpönten, Steuergestaltung Gebrauch zu machen. ${ }^{81}$ Die Pflicht des Vorstands zum Reputationsmanagement stellt freilich keine Einbahnstraße dar, sondern kann es umgekehrt auch erfordern, Handlungen zu unterlassen, die der Unternehmensreputation abträglich

Hopt/M. Roth in Großkomm/AktG, 5. Aufl. 2015, § 93 AktG Rz. 210; Windmöller in FS Budde, 1995, S. 675, 679.

Baas, Leitungsmacht und Gemeinwohlbindung der AG, 1976, S. 211; ablehnend Fleischer, AG 2001, 171, 178.

BGH v. 6.12.2001 - 1 StR 215/01, BGHSt 47, 187, $197=$ AG 2002, 347, 349 f.; Götze/Bicker in Krieger/Uwe H. Schneider, Hdb. Managerhaftung, 3. Aufl. 2017, § 30 Rz. 106; Uwe H. Schneider, AG 1983, 205, $212 \mathrm{f}$. 
sind. ${ }^{82}$ Darüber wie sich diese Pflicht im Einzelfall konkretisiert, bestehen allerdings noch einige Unsicherheiten. ${ }^{83}$

Eine Reputationsproblematik eröffnet sich insbesondere bei Spenden an radikale Parteien, die einen massiven Verlust der Gesellschaft im öffentlichen Ansehen nach sich ziehen können. Das illustriert der Fall der Unternehmensgruppe Theo Müller, über dessen Namensgeber sich hartnäckig das Gerücht hält, er spende an die NPD. Obwohl dies erwiesenermaßen nicht zutrifft, hat das Unternehmen von auf dieser Behauptung fußenden Boykottaufrufen nachhaltig wirtschaftlichen Schaden genommen. ${ }^{84}$ Jedenfalls Spenden an verfassungswidrige Parteien verbieten sich von daher ${ }^{85}$ Aber auch Spenden an sonstige Parteien an den politischen Rändern, die noch nicht die hohen Voraussetzungen des Art. 21 Abs. 2 GG erfüllen, aber allgemein auf erhebliche soziale Ablehnung stoßen, wirken sich reputationsschädigend aus und sind daher zu unterlassen. ${ }^{86}$

Bei Hege und Pflege der Unternehmensreputation stellt sich ferner die Frage, wie mit Parteispenden zu verfahren ist, die geeignet sind, öffentlich den Eindruck zu erwecken, die Gesellschaft versuche sich konkrete Vorteile zu erkaufen. Über das Maß hinaus, das bereits die Legalitätspflicht dem Vorstand abverlangt, ${ }^{87}$ ist es ratsam, schon den bösen Schein zu vermeiden und sich bei der Spendenvergabe eine gewisse Zurückhaltung aufzuerlegen ${ }^{88}$ - insbesondere dann, wenn in Angelegenheiten, die unmittelbar die geschäftliche Betätigung berühren, das politische Klima angespannt ist und Wahlen unmittelbar bevorstehen. Das sich selbst in solchen Situationen Spenden aber als pflichtwidrig darstellen, wird eher die Ausnahme als die Regel bilden. Schließlich ist auch hier dem Vorstand ein unternehmerisches Ermessen zu gewähren, in dessen Rahmen er die erwarteten Vorteile der politischen „Landschaftspflege“ mit einem etwaigen Reputationsverlust abzuwägen hat. ${ }^{89}$

\section{Legalitätspflicht}

Ferner muss der Vorstand bei der Spendenvergabe seiner Legalitätspflicht gerecht werden. Diese gebietet es dem Vorstand nicht nur, die innergesellschaftliche Zuständigkeitsordnung zu wahren (interne Pflichtenbindung), ${ }^{90}$ sondern auch sämtliche Rechtsnormen zu befolgen, die das Unternehmen im Außenverhältnis als Rechtssubjekt treffen (externe Pflichtenbindung). ${ }^{91}$

\section{a) Parteiengesetz}

Es mag zunächst naheliegend erscheinen, dass der Vorstand aufgrund seiner Legalitätspflicht, die Verbote des $\S 25$ Abs. 2 ParteiG zu beachten hat. Das offenbart sich bei

Seibt, DB 2015, 171, 175; Fleischer, DB 2017, 2015, 2021.

Fleischer, AG 2017, 509, 516: „Seine rechtssatzförmige Verdichtung steht allerdings noch aus.“

Siehe Walter, „Müller Milch und NPD: Wenn Gerüchte Millionen kosten“, news.de, 8.6.2012, abrufbar unter: www.news.de/wirtschaft/ 855314590/mueller-milch-und-die-npd-wenn-geruechtemillionen-kos ten/1/ (zuletzt abgerufen am 8.6.2018).

Vgl. Uwe H. Schneider, AG 1983, 205, 212.

Vgl. H. P. Westermann, ZIP 1990, 771, 777 mit dem Beispiel einer Spende an die Partei „Die Republikaner".

Siehe dazu IV.2.b).

Götze/Bicker in Krieger/Uwe H. Schneider, Hdb. Managerhaftung, 3. Aufl. 2017, § 30 Rz. 107; Mertens/Cahn in KölnKomm/AktG, 3. Aufl. 2010, § 76 AktG Rz. 41; Kind, NZG 2000, 567, 569 f.; Kort in Großkomm/AktG, 5. Aufl. 2015, § 76 AktG Rz. 111.

Vgl. Seibt in K. Schmidt/Lutter, 3. Aufl. 2015, § 76 AktG Rz. 24.

Fleischer in Spindler/Stilz, 3. Aufl. 2015, § 93 AktG Rz. 20.

Spindler in MünchKomm/AktG, 4. Aufl. 2014, § 93 AktG Rz. 74. 
näherem Hinsehen aber als Trugschluss, da Normadressat dieser Vorschriften ausschließlich die Partei, nicht aber der Spender ist. Das zeigt sich nicht nur am Wortlaut der Vorschrift („Von der Befugnis der Parteien, Spenden anzunehmen ausgeschlossen sind $\left.[\ldots]^{\prime \prime}\right)$, sondern auch an ihren Rechtsfolgen: Unzulässige Spenden hat die Partei an den Bundestagspräsidenten weiterzuleiten ( $§ 25$ Abs. 4 ParteiG), für Verstöße wird nur sie sanktioniert (§ 31c ParteiG). Der Vorstand verletzt darum nicht schon deshalb seine Legalitätspflicht, weil er eine Spende tätigt, die nach dem ParteiG unzulässig ist ${ }^{92}-$ etwa da die Gesellschaft überwiegend im Eigentum der öffentlichen Hand steht ( $§ 25$ Abs. 2 Nr. 5 ParteiG).

$\S 25$ Abs. 2 ParteiG wirkt allerdings auf die allgemeine Sorgfaltspflicht des Vorstands zurück. Spenden, die die Partei nicht annehmen darf, verfehlen ihren Zweck. Ist für den Vorstand ex ante zu erkennen, dass die Partei verpflichtet ist, die Spende an den Bundestagspräsidenten weiterzuleiten, verhält er sich darum pflichtwidrig. ${ }^{93}$

\section{b) Korruptionsstrafrecht}

Der Vorstand verletzt jedoch seine Legalitätspflicht, wenn er mit der Spendengewährung eine Straftat begeht. ${ }^{94}$ Dabei sind insbesondere die Straftatbestände der Vorteilsgewährung an Amtsträger (§ 333 Abs. 1 StGB) und der Bestechung von Mandatsträgern ( $\$ 108$ e Abs. 2 StGB) ins Auge zu fassen. ${ }^{95}$ Zwar ist die Partei selbst weder Amtsträger (§ 11 Abs. 1 Nr. 2 StGB) noch Mitglied einer Volksvertretungen ( $\$ 108$ e Abs. 1, 3 StGB), doch lassen es beide Tatbestände genügen, dass einem Dritten ein Vorteil angeboten, versprochen oder gewährt wird. Sie umfassen damit auch Konstellationen, in denen die Parteispende eine Gegenleistung für die Tätigkeit eines Regierungsmitglieds oder Abgeordneten darstellt. ${ }^{96}$ Für die Vorteilsgewährung genügt es bereits, dass die Parteispende ein funktionales Äquivalent zur Amtsträgertätigkeit bildet (sog. Unrechtsvereinbarung). ${ }^{97}$ Geringfügig strengere Anforderungen stellt der Tat-

480 bestand der Mandatsträgerbestechung, der verlangt, dass eine bestimmte Handlung des Mandatsträgers die Gegenleistung für die Spende bildet (sog. konkrete Unrechtsvereinbarung). ${ }^{98}$ Nur scheinbar einen sicheren Hafen bietet $\S 108$ e Abs. 4 Satz 2 StGB dem Vorstandsmitglied. Danach erfasst der Tatbestand Spenden, die nach dem ParteiG zulässig sind, nicht. Spenden, die in Erwartung einer politischen Gegenleistung gewährt werden, erklärt $\S 25$ Abs. 2 Nr. 7 ParteiG aber gerade für unzulässig.

Anschauungsmaterial dazu, wie schnell Parteispenden den Verdacht einer Straftat begründen können, bietet derzeit die H\&K AG. Das Unternehmen sieht sich jüngst mit dem Vorwurf konfrontiert, Mitarbeiter hätten gezielt Spenden an mehrere Parteien und Bundestagsabgeordnete getätigt, um eine Ausfuhrgenehmigung für das Sturmgewehr G36 nach Mexiko zu erhalten. ${ }^{99}$

Siehe auch Baas, Leitungsmacht und Gemeinwohlbindung der AG, 1976, S. 212.

Vgl. Zachert, Grenzen des unternehmerischen Ermessens bei der Vergabe von Unternehmensspenden im US-amerikanischen Gesellschafts- und im deutschen Kapitalgesellschaftsrecht, 2005, S. 200. Vgl. statt aller Hopt/M. Roth in Großkomm/AktG, 5. Aufl. 2015, § 93 AktG Rz. 141.

Siehe auch Säcker, BB 2009, 282, 283 f.; Uwe H. Schneider, AG 1983, 205, 212. Vgl. H. E. Müller in MünchKomm/StGB, 3. Aufl. 2017, § 108e StGB Rz. 25.

Fischer, 65. Aufl. 2018, § 331 StGB Rz. 21; Heine/Eisele in Schönke/Schröder, 29. Aufl. 2014, $\S 331$ StGB Rz. 36.

H. E. Müller in MünchKomm/StGB, 3. Aufl. 2017, § 108e StGB Rz. 36 f.

Dazu Vogel, „Parteispenden für Waffen“, taz, 22.5.2018, abrufbar unter: http://www.taz.de/!5504160/ (zuletzt abgerufen am 8.6.2018). 


\section{Treuepflicht}

Auch die organschaftliche Treuepflicht zieht dem Vorstand bei der Spendenvergabepraxis Grenzen. Sie gebietet es ihm in allen Angelegenheiten, die das Interesse der Aktiengesellschaft berühren, allein deren Wohl und nicht seinen eigenen Nutzen im Auge zu haben. ${ }^{100}$ Ein Konflikt mit dieser Pflicht ergibt sich, wenn der Vorstand Spenden an Parteien vergibt, in denen er sich auch als Privatperson politisch engagiert. Hier entsteht schnell der Verdacht, dass die Spende durch persönliche Interessen an der eigenen politischen Karriere motiviert ist. ${ }^{101} \mathrm{Um}$ diesen Interessenkonflikt zu vermeiden, wird das betroffene Vorstandsmitglied die Spendenentscheidung nicht alleine fällen dürfen. ${ }^{102}$

\section{Fazit}

1. Parteispenden durch Aktiengesellschaft stellen sich sowohl im Außen- als auch im Innenverhältnis als besonders konfliktträchtig dar. Im Außenverhältnis sind Parteispenden von Aktiengesellschaften aufgrund ihrer wirtschaftlichen Stärke im besonderen Maße geeignet, den politischen Wettbewerb einseitig zu verzerren und den Eindruck zu erwecken, die Politik lasse sich von Sonderinteressen beeinflussen. Im Innenverhältnis rührt der Konflikt daher, dass Parteispenden dem Erwerbszweck der Gesellschaft zumindest nicht unmittelbar zu Gute kommen und Vorstand und Aktionäre möglicherweise verschiedene politische Präferenzen aufweisen.

2. Aktiengesellschaften genießen ein Artt. 5 Abs. 1 Satz 1, 19 Abs. 3 GG entspringendes subjektives Recht, an politische Parteien zu spenden. Dieses unterliegt der Schranke des qualifizierten Gesetzesvorbehaltes (Art. 5 Abs. 2 GG) und kann im Hinblick auf den Schutz anderer Verfassungsgüter weitreichenden Beschränkungen unterworfen werden. Adressat der öffentlich-rechtlichen Regulierung der Parteispende ist bislang allerdings vor allem die Partei, nicht die Gesellschaft.

3. Im Binnenverhältnis ist der Vorstand im Regelfall allein dafür zuständig, über Parteispenden aus dem Gesellschaftsvermögen zu beschließen. Er unterliegt keiner politischen Neutralitätspflicht, hat aber gegebenenfalls ein von einem Code of Conduct ausgehendes Spendenverbot oder einen Zustimmungsvorbehalt zugunsten des Aufsichtsrates zu berücksichtigen.

4. Rechtspolitisch bedarf es einer weiteren Debatte darüber, ob eine Zustimmungskompetenz der Hauptversammlung für Parteispenden aus dem Gesellschaftsvermögen nach dem Vorbild des s 366 Companies Act 2006 eingeführt werden sollte.

5. Tätigt der Vorstand Spenden aus dem Gesellschaftsvermögen, hat er dabei einige Vorgaben zu beachten, die sich aus seiner Sorgfalts- und Treuepflicht ergeben. Insbesondere im Zusammenhang mit kontroversen politischen Entscheidungen, die das Unternehmensinteresse berühren, ist Vorsicht geboten. 